\title{
Analytic Formulation for the Sound Absorption of a Panel Absorber under the Effects of Microperforation, Air Pumping, Linear Vibration and Nonlinear Vibration
}

\author{
Y.Y. Lee \\ Department of Civil and Architectural Engineering, City University of Hong Kong, Kowloon Tong, Kowloon, Hong Kong \\ Correspondence should be addressed to Y. Y. Lee; bcraylee@cityu.edu.hk
}

Received 27 March 2014; Revised 24 June 2014; Accepted 1 July 2014; Published 23 July 2014

Academic Editor: Rehana Naz

Copyright (C) 2014 Y. Y. Lee. This is an open access article distributed under the Creative Commons Attribution License, which permits unrestricted use, distribution, and reproduction in any medium, provided the original work is properly cited.

\begin{abstract}
This study includes the first work about the absorption of a panel absorber under the effects of microperforation, air pumping, and linear and nonlinear vibrations. In practice, thin perforated panel absorber is backed by a flexible wall to enhance the acoustic performance within the room. The panel is easily excited to vibrate nonlinearly and the wall can vibrate linearly. However, the assumptions of linear panel vibration and no wall vibration are adopted in many research works. The development of the absorption formula is based on the classical approach and the electroacoustic analogy, in which the impedances of microperforation, air pumping, and linear and nonlinear vibrations are in parallel and connected to that of the air cavity in series. Unlike those finite element, numerical integration, and multiscale solution methods and so forth, the analytic formula to calculate the absorption of a panel absorber does not require heavy computation effort and is suitable for engineering calculation purpose. The theoretical result obtained from the proposed method shows reasonable agreement with that from a previous numerical integration method. It can be concluded that the overall absorption bandwidth of a panel absorber with an appropriate configuration can be optimized and widened by making use of the positive effects of microperforation, air pumping, and panel vibration.
\end{abstract}

\section{Introduction}

Numerous studies of structural-acoustic interaction have been carried out in recent decades (e.g., [1-6]). Particularly, the topics of microperforated panel and panel absorber have attracted the interest of many researchers. It is because (1) microperforated panel and panel absorber can be made of any durable materials, (2) their maintenance is relatively simple, and (3) microperforated panel requires small space to achieve high sound absorption when compared with typical foam or porous materials. Acoustic theories for these two treatments were developed by Mulholland and Parbrook [7], Ford and McCormick [8], and Maa [9, 10]. According to the assumptions of the previous studies, the wall behind a panel absorber was considered as rigid panel (i.e., no vibration) and the panel absorber was considered to vibrate linearly (in fact, thin panels are easy to vibrate nonlinearly). Although many linear structural-acoustic or nonlinear structural vibration problems had been solved (e.g. [11-17]), very limited studies considered the nonlinear vibration effect on the sound absorption performance of a panel absorber. In fact, it is more appropriate to employ nonlinear approach to analyze the vibration of a thin panel. There are five of those limited nonlinear structural-acoustic research works [18-22], which focused on the natural frequencies of nonlinear structuralacoustic systems, and the sound absorption, radiation, and transmission of nonlinear flat/curved panels. These works are relevant to the nonlinear structural-acoustic problem handled in this paper, but the researchers adopted the solution methods which required heavy computational efforts (e.g., finite element method, numerical integration method, and harmonic balance method). Other solution methods for large amplitude structural vibrations and nonlinear oscillations (e.g., the method of multiple scales, the method of normal forms, the method of Shaw and Pierre, and the method of King and Vakakis [23-27]) also require relatively tedious solution implementation and heavy computational efforts. In [28], the simple and straightforward electroacoustic analogy 
was adopted for modelling of the sound absorption of a panel absorber. Besides, the air pumping effect (or Helmholtz resonance effect) has been mentioned in many textbooks and articles (e.g., [29]), but it has not been incorporated into the research problem of nonlinear panel absorber. In this paper, it focuses on the development of the analytic absorption formula of a panel absorber which considers the four effects (i.e., microperforation, air pumping, and linear and nonlinear vibrations).

\section{Analytic Formulation}

2.1. Nonlinear Panel Vibration and Liner Wall Vibration. Figure 1 shows the proposed panel absorber subject to the effects of microperforation, air pumping, and linear and nonlinear vibrations. Firstly, the impedances of the nonlinear panel and linear wall are derived in this subsection. According to the formulation of Chu and Herrmann [30], the governing equation for the nonlinear vibration of a rectangular panel subject to uniformly distributed harmonic excitation can be expressed in the following form (see Appendix A for more):

$$
\rho \frac{d^{2} \varepsilon}{d \tau^{2}}+\rho \omega_{o}^{2} \varepsilon+\beta \varepsilon^{3}+F(\tau)=0
$$

where

$F(\tau)=F_{o, m n} \sin (\omega \tau)=$ modal harmonic excitation,

$F_{o, m n}=F_{o} \Lambda_{m n}=\lambda \rho t g \Lambda_{m n}=$ modal excitation magnitude,

$F_{o}=\lambda \rho t g=$ physical excitation magnitude,

$g=$ gravity acceleration $(9.81 \mathrm{~ms}-2)$,

$\tau=$ time,

$\lambda=$ dimensionless excitation parameter,

$\Lambda_{m n}=\frac{\left(\int_{0}^{b} \int_{0}^{a} \sin (m \pi x / a) \sin (n \pi y / b) d x d y\right)}{\int_{0}^{b} \int_{0}^{a}[\sin (m \pi x / a) \sin (n \pi y / b)]^{2} d x d y}$

$=$ modal coefficient,

$\omega=$ excitation frequency,

$\omega_{o}=$ linear resonant frequency of the $(m, n)$ mode,

$m, n=$ structural mode numbers,

$$
\begin{aligned}
\beta= & \frac{E t}{12\left(1-v^{2}\right) a^{4}} \frac{3 \pi^{4}}{2} \\
\times & {\left[\frac{1}{2} m^{2}\left(m^{2}+v n^{2} r^{2}\right)\left(-\frac{3}{2}+v \frac{n^{2} r^{2}}{m^{2}}\right)\right.} \\
& \quad-\frac{3}{4} m^{2}\left(m^{2}+v n^{2} r^{2}\right)
\end{aligned}
$$

$$
\begin{aligned}
& +\frac{1}{2} n^{2} r^{2}\left(n^{2} r^{2}+v m^{2}\right)\left(-\frac{3}{2}+v \frac{m^{2}}{n^{2} r^{2}}\right) \\
& \left.-\frac{3}{4} n^{2} r^{2}\left(n^{2} r^{2}+v m^{2}\right)\right]
\end{aligned}
$$

$=$ nonlinear stiffness coefficient,

$r=a / b$ is the aspect ratio; $E$ is Young's modulus; $\rho$ is the surface density; $v$ is Poisson's ratio; and $t, a$, and $b$ are the thickness, length, and width.

Consider the approximation of $\varepsilon=A \cos (\omega \tau)$ in (1). Then

$$
\frac{3}{4} \beta A^{3}+\rho\left(\omega_{o}^{2}-\omega^{2}\right) A+F_{o, m n}=0
$$

$\Rightarrow$

$$
A^{3}+\frac{4}{3 \beta} \rho\left(\omega_{o}^{2}-\omega^{2}\right) A+\frac{4}{3 \beta} F_{o, m n}=0
$$

where $A=$ modal vibration amplitude.

For simplicity, let $p=(4 / 3 \beta) \rho\left(\omega_{o}^{2}-\omega^{2}\right), q=(4 / 3 \beta) F_{o, m n}$, and $A=B-(p / 3 B)$ (i.e., Vieta's substitution [31]). Equation (5) can be rewritten as

$$
B^{3}+q-\frac{p^{3}}{27 B^{3}}=0
$$

$\Rightarrow$

$$
B^{6}+q B^{3}-\frac{p^{3}}{27}=0
$$

If $B^{3}$ is considered as an independent unknown, (7) is a "modified" quadratic equation. Then, the analytic formulas for the cubic solutions are given below:

$$
\begin{aligned}
& A_{1}=-\frac{1}{3} \sqrt[3]{\frac{1}{2}(\Theta+\Omega)}-\frac{1}{3} \sqrt[3]{\frac{1}{2}(\Theta-\Omega)} \\
& A_{2}=\frac{1+j \sqrt{3}}{6} \sqrt[3]{\frac{1}{2}(\Theta+\Omega)}+\frac{1-j \sqrt{3}}{6} \sqrt[3]{\frac{1}{2}(\Theta-\Omega)} \\
& A_{3}=\frac{1-j \sqrt{3}}{6} \sqrt[3]{\frac{1}{2}(\Theta+\Omega)}+\frac{1+j \sqrt{3}}{6} \sqrt[3]{\frac{1}{2}(\Theta-\Omega)},
\end{aligned}
$$

where $\Theta=\sqrt{(27 q)^{2}+4(3 p)^{3}}, \Omega=27 q$.

In the next step, the nonlinear stiffness can be defined as $(3 / 4) \beta A^{2}$ and overall panel stiffness is $\rho \omega_{o}^{2}+(3 / 4) \beta A^{2}$. The panel impedance of the $(m, n)$ mode is given by

$$
Z_{P, m n}=\frac{F_{o, m n}}{V_{P, m n}}=\frac{2 \rho \xi \omega \omega_{N}+j\left[\rho\left(\omega^{2}-\omega_{o}^{2}\right)-(3 / 4) \beta A^{2}\right]}{\omega},
$$

where $j=\sqrt{-1}=$ complex number; $\xi$ is the panel damping ratio. $V_{P, m n}$ is the modal panel velocity. Note that $\omega_{N}$ is 


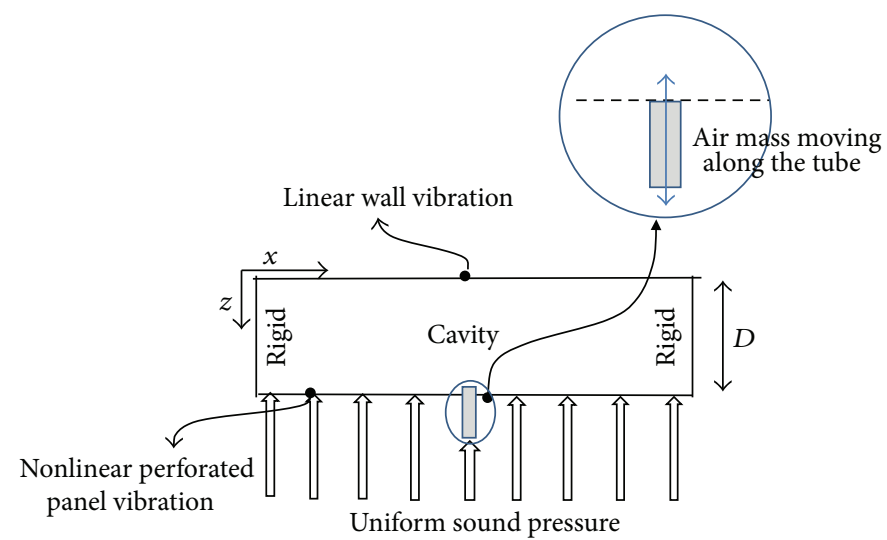

FIGURE 1: A panel absorber subject to the effects of microperforation, air pumping, and linear and nonlinear vibrations.

the nonlinear peak frequency, instead of the linear natural frequency, $\omega_{o}$ in [32] (see Appendix B for the derivation of $\left.\omega_{N}\right)$. When it is the case of small amplitude vibration or linear vibration, $\omega_{N}$ is equal to $\omega_{0}$.

Consider the overall panel velocity contributed from the modal velocities and find the overall panel impedance:

$$
V_{P}=\sum_{m}^{M} \sum_{n}^{N} \frac{F_{o} \Lambda_{m n} \Lambda_{m n}^{\prime}}{Z_{P, m n}}
$$

$\Rightarrow$

$$
Z_{P}=\frac{F_{o}}{V_{P}}=\left(\sum_{m}^{M} \sum_{n}^{N} \frac{\Lambda_{m n} \Lambda_{m n}^{\prime}}{Z_{P, m n}}\right)^{-1},
$$

where $\Lambda_{m n}^{\prime}=\left(\int_{0}^{b} \int_{0}^{a} \sin (m \pi x / a) \sin (n \pi y / b) d x d y\right) / a b$. According to [33], the sound absorption of two linearly vibrating plates with different boundary conditions was very close. It was concluded that the boundary condition would not affect the sound absorption significantly. That is why only one boundary condition case is considered here.

Consider the linear case (or small amplitude case) in the model representing the panel vibration and the corresponding modal and overall panel impedances (i.e., (9), (11)). It is noted that, for the linear vibration, the fundamental harmonic balance can lead to the exact solution (no higher harmonic terms are required). Hence the modal and overall wall impedances are given below:

$$
\begin{aligned}
Z_{W, m n} & =\frac{2 \rho_{W} \xi_{W} \omega \omega_{W}+j\left[\rho\left(\omega^{2}-\omega_{W}^{2}\right)\right]}{\omega}, \\
Z_{W} & =\left(\sum_{m}^{M} \sum_{n}^{N} \frac{\Lambda_{m n} \Lambda_{m n}^{\prime}}{Z_{W, m n}}\right)^{-1},
\end{aligned}
$$

where $\xi_{W}, \rho_{W}$, and $\omega_{W}$ are the wall damping factor, density, and natural frequency

2.2. Air Pumping (Helmholtz Resonance). In Figure 1, the impedance of the air pumping along the tube mounted on the panel absorber is obtained here. The differential equation for the air mass pumping in/out along the tube is given by [29]

$$
\rho_{a} L^{\prime} \frac{d^{2} \Lambda}{d \tau^{2}}+\frac{\left(R_{r}+R_{w}\right)}{S} \frac{d \Lambda}{d \tau}+P(\tau)=0
$$

where $\Lambda=$ air mass displacement, $P(\tau)=$ acoustic pressure excitation, $R_{r}=\rho_{a} c_{a} k^{2} S^{2} / 4 \pi=$ damping due to radiation resistance, $R_{w}=2 \rho_{a} L^{\prime} S \alpha_{w}=$ damping due to thermoviscous resistance, $L^{\prime}=L+1.4 a_{t}=$ effective tube length, $\alpha_{w}=$ $\left(1 / a_{t} c_{a}\right)\left(\eta \omega / 2 \rho_{a}\right)^{0.5}(1+(\gamma / \sqrt{\operatorname{Pr}}))=$ absorption coefficient for wall losses, and $\rho_{a}$ is the air density; $L^{\prime}$ is the effective length of the tube with unflanged outer end; $k$ is the wave number; $L$ is the tube length; $a_{t}$ is the radius of the tube; $S$ is the cross section area of the tube; $\eta$ is the air viscosity; $\gamma$ is the ratio of specific heat; $\operatorname{Pr}$ is Prandtl number.

Similar to the overall panel impedance in (9), the impedance of air pumping along the tube is given below:

$$
Z_{H}^{\prime}=\frac{\left(\left(R_{r}+R_{w}\right) / S\right) \omega+j \rho_{a} L^{\prime} \omega^{2}}{\omega} .
$$

Using (15), the air mass velocity is given by

$$
Z_{H}^{\prime}=\frac{P_{o}}{V_{H}^{\prime}} \Longrightarrow V_{H}^{\prime}=\frac{P_{o}}{Z_{H}^{\prime}}
$$

where $P_{o}=$ acoustic pressure magnitude.

Consider the average velocity over the panel surface

$$
V_{H}=V_{H}^{\prime} \sigma_{H},
$$

where $\sigma_{H}=$ the ratio of tube area to panel area.

Then the overall impedance of air pumping is obtained by substituting (17) into (16):

$$
Z_{H}=\frac{Z_{H}^{\prime}}{\sigma_{H}} .
$$

2.3. Microperforation. In Figure 1, the impedance of the microholes over the panel surface is obtained here. According 
to $[9,10]$, the real and imaginary parts of the acoustic impedance of a microhole are given below:

$$
\begin{aligned}
& Z_{M, R}^{\prime}=\rho_{a} c_{a}\left(0.147 \frac{t}{d^{2}}\right)\left(\sqrt{9+\frac{100 d^{2} f}{32}}+1.768 \sqrt{f} \frac{d^{2}}{t}\right), \\
& Z_{M, I}^{\prime}=\rho_{a} c_{a} 1.847 f t\left(1+\frac{1}{\sqrt{9+50 d^{2} f}}+0.85 \frac{d}{t}\right),
\end{aligned}
$$

where $Z_{M}^{\prime}=Z_{M, R}^{\prime}+j Z_{M, I}^{\prime}=$ the impedance of a microhole, $f$ is frequency, $t$ is panel thickness, and $d$ is the perforation diameter. $\rho_{a}$ and $c_{a}$ are air density and sound speed.

Then, the overall impedance of all microholes over the panel is given by

$$
Z_{M}=\frac{Z_{M}^{\prime}}{\sigma}
$$

where $\sigma$ is the perforation ratio.

2.4. Air Cavity. In Figure 1, the impedance of the air cavity is obtained here. The acoustic velocity potential within a rectangular cavity is given by the following homogeneous wave equation [1]:

$$
\nabla^{2} \phi-\frac{1}{c_{a}^{2}} \frac{\partial^{2} \phi}{\partial \tau^{2}}=0
$$

where $\phi$ is the velocity potential function.

The air particle velocities in the $x, y$, and $z$ directions and pressures within the air cavity can be given by $\partial \phi / \partial x, \partial \phi / \partial y, \partial \phi / \partial z$, and $-\rho_{a}(\partial \phi / \partial \tau)$, respectively.

The boundary conditions of the rectangular cavity to be satisfied are

$$
\begin{aligned}
& \left.\frac{\partial \phi}{\partial x}\right|_{x=0}=\left.\frac{\partial \phi}{\partial x}\right|_{x=a}=0 \\
& \left.\frac{\partial \phi}{\partial y}\right|_{y=0}=\left.\frac{\partial \phi}{\partial y}\right|_{y=b}=0 \\
& \left.\frac{\partial \phi}{\partial z}\right|_{z=0}=0 \\
& \left.\frac{\partial \phi}{\partial z}\right|_{z=-D}=V_{D}
\end{aligned}
$$

where $a$ and $b$ are the length and width of the rectangular cavity or the panel and $V_{D}$ is the average velocity at $z=$ $D=$ cavity depth. Note that the cavity impedance of no wall vibration is derived here and used into the sound absorption formulation in the next subsection which can consider both the panel and wall vibration effects and is derived using the electroacoustic analogy.
By applying the boundary conditions in (22a) and (22b), the solution of (21) can be expressed as

$$
\begin{aligned}
\phi= & \sum_{u=0}^{U} \sum_{w=0}^{W}\left[L_{u w} \cosh \left(\mu_{u w} z\right)+N_{u w} \sinh \left(\mu_{u w} z\right)\right] \\
& \times \cos \left(\frac{u \pi x}{a}\right) \cos \left(\frac{w \pi y}{b}\right) e^{i \omega \tau}
\end{aligned}
$$

where $\mu_{u w}=\sqrt{\left((u \pi / a)^{2}+(w \pi / b)^{2}\right)-\left(\omega / c_{a}\right)^{2}}, u$ and $w$ are the acoustic mode numbers, $U$ and $W$ are the numbers of acoustic modes used in the $x$ and $y$ directions, and $L_{u w}$ and $N_{u w}$ are coefficients to be determined in the following step.

Substituting (23) into (22c) and (22d) gives

$$
\begin{gathered}
\left.\frac{\partial \phi}{\partial z}\right|_{z=0}=0 \Longrightarrow N_{u w}=0 \\
\left.\frac{\partial \phi}{\partial z}\right|_{z=D}=V_{D} e^{i \omega \tau} \Longrightarrow L_{u w} \\
=-\frac{\int_{0}^{a} \int_{0}^{b} V_{D} \cos (u \pi x / a) \cos (w \pi y / b) d x d y}{\kappa_{u w} \mu_{u w} \sinh \left(\mu_{u w} D\right)}
\end{gathered}
$$

where $\kappa_{u w}=\int_{0}^{a} \int_{0}^{b} \cos (u \pi x / a)^{2} \cos (w \pi y / b)^{2} d x d y$.

By inputting (24a) and (24b) into (23), the pressure at $z=$ $D$ is obtained and given below:

$$
\begin{aligned}
& F_{D} \\
& \begin{aligned}
&=\sum_{u=0}^{U} \sum_{w=0}^{W}[ j \rho_{a} \omega \frac{\operatorname{coth}\left(\mu_{u w} D\right)}{\kappa_{u w} \mu_{u w}} \\
&\left.\times \int_{0}^{a} \int_{0}^{b} V_{D} \cos \left(\frac{u \pi x}{a}\right) \cos \left(\frac{w \pi y}{b}\right) d x d y\right] \\
& \times \cos \left(\frac{u \pi x}{a}\right) \cos \left(\frac{w \pi y}{b}\right) .
\end{aligned}
\end{aligned}
$$

The average pressure force at $z=D$ is then given by taking integration over the panel area

$$
\begin{aligned}
\bar{F}_{D} & =\frac{\int_{0}^{a} \int_{0}^{b} F_{D} \cos (u \pi x / a) \cos (w \pi y / b) d x d y}{a b} \\
& =V_{D} j \rho_{a} \omega \sum_{u=0}^{U} \sum_{w=0}^{W}\left[\frac{\kappa_{u w}^{\prime}}{\kappa_{u w}} \frac{\operatorname{coth}\left(\mu_{u w} D\right)}{\mu_{u w}}\right],
\end{aligned}
$$

where $\kappa_{u w}^{\prime}=\left(\int_{0}^{a} \int_{0}^{b} \cos (u \pi x / a) \cos (w \pi y / b) d x d y\right)^{2} / a b$.

Finally, the impedance of the air cavity is given by

$$
Z_{\mathrm{cav}}=\frac{\bar{F}_{D}}{V_{D}}=j \rho_{a} \omega \sum_{u=0}^{U} \sum_{w=0}^{W}\left[\frac{\kappa_{u w}^{\prime}}{\kappa_{u w}} \frac{\operatorname{coth}\left(\mu_{u w} D\right)}{\mu_{u w}}\right] .
$$

Note that since even pressure distribution is considered, then $u, w=0,2,4, \ldots$ If $a$ and $b$ are less than $0.3 \mathrm{~m}$, then the 
frequencies of the fundamental resonances parallel to the cavity length or width are higher than $1000 \mathrm{~Hz}$ (note that $1000 \mathrm{~Hz}$ is not within the frequency range concerned in this study). Then, in this case, only the mode numbers, $u=0$ and $w=0$, are considered. The impedance can be approximated by

$$
Z_{\mathrm{cav}} \approx-j \rho_{a} \omega \cot \left(\frac{\omega}{c_{a}} D\right)
$$

2.5. Electroacoustic Analogy. According to [34], the acoustic impedance of a double panel absorber can be analogous to that of the electrical circuit. The acoustic impedance is given below:

$$
Z_{\mathrm{dou}}=Z_{p, 1}+Z_{\mathrm{cav}, 1}+\frac{\left(Z_{\mathrm{cav}, 1}\right)^{2}}{Z_{p, 2}+Z_{\mathrm{cav}, 1}+Z_{\mathrm{cav}, 2}},
$$

where $Z_{p, 1}$ and $Z_{p, 2}=$ acoustic impedance of the 1st and 2 nd panels; $Z_{\text {cav }, 1}$ and $Z_{\text {cav }, 2}=$ acoustic impedance of the 1st and 2nd cavities.

If the impedance of the 2nd cavity is set to zero, that is, equivalent to the case of a panel absorber backed by a flexible wall, the overall impedance in (29) is rewritten as

$$
Z_{o}=Z_{\mathrm{com}}+Z_{\mathrm{cav}}+\frac{\left(Z_{\mathrm{cav}}\right)^{2}}{Z_{W}+Z_{\mathrm{cav}}},
$$

where $Z_{\text {com }}=\left(Z_{M} Z_{P} Z_{H}\right) /\left(Z_{M} Z_{P}+Z_{P} Z_{H}+Z_{H} Z_{M}\right)=$ combined impedance. $Z_{M}$ = impedance of the microperforation; $Z_{P}=$ acoustic impedance of the panel absorber; $Z_{H}=$ impedance of the air pumping; $Z_{W}=$ impedance of the wall.

If the wall is perfectly rigid (i.e., no vibration), the natural frequency of wall $\omega_{W}$ in $(12) \rightarrow \infty$. Hence, $Z_{W, m n}, Z_{W} \rightarrow \infty$ and $\left(Z_{\text {cav }}\right)^{2} /\left(Z_{W}+Z_{\text {cav }}\right) \approx 0$. Equation (30) can be rewritten as

$$
Z_{o}=Z_{\text {com }}+Z_{\text {cav }}
$$

Then the absorption coefficient of the absorber $\alpha_{o}$ for normal incidence can be calculated by the well-known formula (see [29]):

$$
\alpha_{o}=\frac{4 \operatorname{Re}\left(Z_{o}\right)}{\left(1+\operatorname{Re}\left(Z_{o}\right)\right)^{2}+\left(\operatorname{Im}\left(Z_{o}\right)\right)^{2}} .
$$

\section{Results and Discussions}

Using (32), the sound absorption is obtained for the cases considered in this section. Each panel absorber is made of aluminum panel. The material properties are Young's modulus $E=7 \times 10^{10} \mathrm{~N} / \mathrm{m}^{2}$, Poisson's ratio $v=0.3$, and mass density $\rho=2700 \mathrm{~kg} / \mathrm{m}^{3}$. The sound speed and air density are $340 \mathrm{~m} / \mathrm{s}$ and $1.2 \mathrm{~kg} / \mathrm{m}^{3}$, respectively. Air viscosity $\eta=1.85 \times 10^{-5} \mathrm{~Pa} \cdot \mathrm{s}$; the ratio of specific heat $\gamma=1.402$; Prandtl number $\operatorname{Pr}=0.71$. Various excitation levels, damping factors, panel sizes, and so forth are considered and their effects on the sound absorption performances are studied in the following paragraphs.

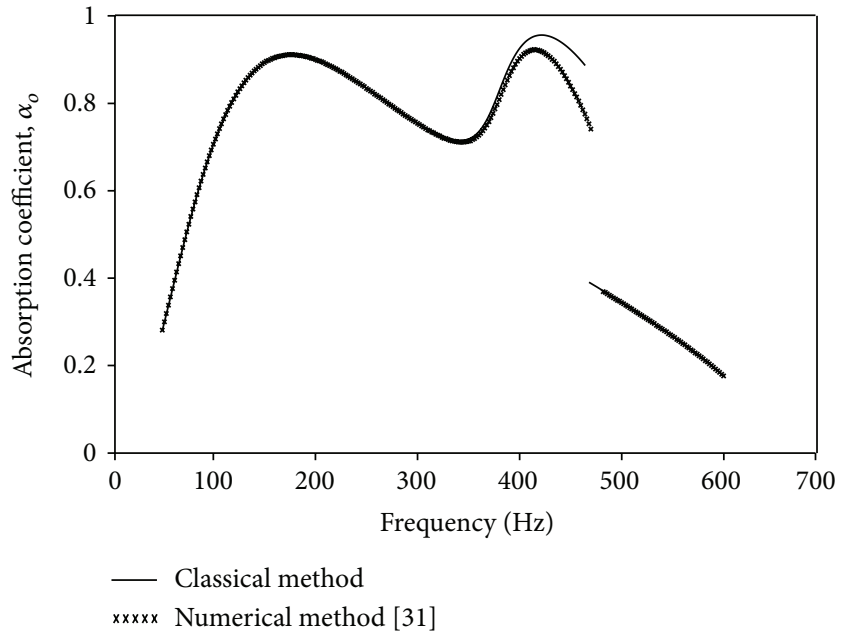

FIGURE 2: Comparison between the results from the proposed classical and numerical methods $(t=0.8 \mathrm{~mm}, a=b=0.1 \mathrm{~m}$, $D=250 \mathrm{~mm}, \xi=0.01, \sigma=0.5 \%, d=0.4 \mathrm{~mm}$, and $\kappa=5.8)$.

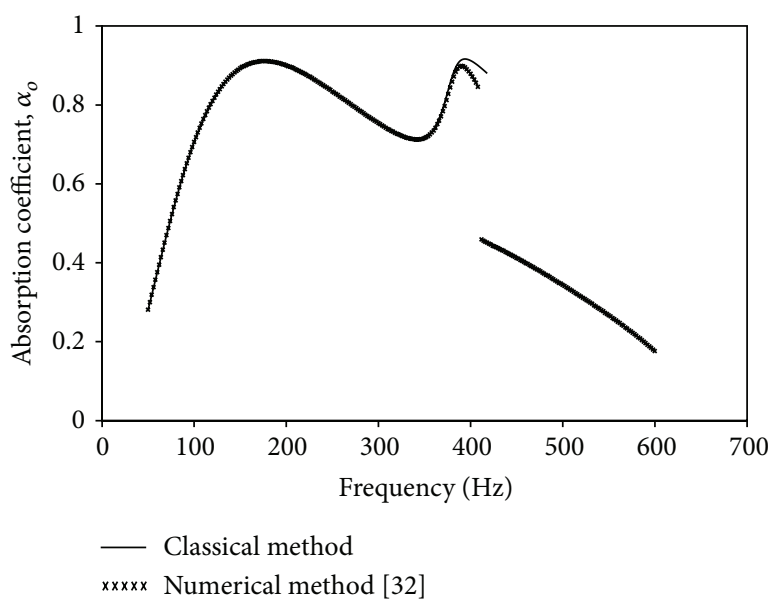

FIGURE 3: Comparison between the results from the proposed classical and numerical methods $(t=0.8 \mathrm{~mm}, a=b=0.1 \mathrm{~m}$, $D=250 \mathrm{~mm}, \xi=0.01, \sigma=0.5 \%, d=0.4 \mathrm{~mm}$, and $\kappa=2.9$ ).

Figures 2 and 3 present comparisons between the frequency-absorption curves obtained from the proposed method and numerical integration method [32]. The results show that there is an absorption peak due to the microperforation around $178 \mathrm{~Hz}$. The well-known "jump phenomenon" can be seen on both curves. The results obtained from the two methods are generally in reasonably good agreement, although some deviations are observed at the absorption peaks around $422 \mathrm{~Hz}$ in Figure 2 and $390 \mathrm{~Hz}$ in Figure 3. Besides, small differences between the jump frequencies (i.e., 464 and $470 \mathrm{~Hz}$ in Figure 2 and 410 and $420 \mathrm{~Hz}$ in Figure 3) are also found. The deviations are caused by that the damping term in the proposed formulation is proportional to the nonlinear peak frequency $\omega_{N}$, instead of the linear natural frequency $\omega_{o}$, which was adopted in [32]. The excitation level in Figure 3 is lower than that in Figure 2. Therefore, the 
structural vibration in Figure 3 is less nonlinear (i.e., $\omega_{N}$ is closer to $\omega_{o}$ ) and the deviation between the peak values from the two methods is smaller. Note that in [32], the numerical integration method did not deliver the unstable solutions. Therefore, only stable results obtained by the proposed method in Figure 3 are compared with the numerical results in [32]. Besides, the unstable results are not obtainable in practical environments and not very useful for practical engineering purpose.

Figures 4(a)-4(c) present the sound absorption plotted against the excitation frequency for various panel resonant frequencies. In each of these three figures, there are two nonlinear solution types. The "jump up" or "jump down" solution is obtained by sweeping the excitation frequency from "high to low" or "low to high". In Figures 4(a)-4(c), the linear $(1,1)$ mode panel resonant frequencies of the three cases are $387 \mathrm{~Hz}, 172 \mathrm{~Hz}$, and $97 \mathrm{~Hz}$, respectively. The absorption peak frequency due to the microperforation effect is $178 \mathrm{~Hz}$. It can be seen that (1) if the panel resonant frequency is higher than the absorption peak frequency due to the microperforation effect, the panel vibration can enhance and widen the absorption bandwidth and (2) if the panel resonant frequency is close to or lower than the absorption peak frequency, the panel vibration degrades the absorption performance and the peak absorption is deteriorated.

Figures 5(a) and 5(b) present the sound absorption plotted against the excitation frequency for various excitation levels. The "jump down" solutions are shown in Figure 5(a) (i.e., sweeping the excitation frequency from "high to low") while the "jump up" solutions are in Figure 5(b) (i.e., sweeping the excitation frequency from "low to high"). The absorption peak around $178 \mathrm{~Hz}$ is due to the microperforation effect and is not significantly affected by the excitation level. It is noted that in the cases of $\kappa=1$, there is no jump phenomenon as the excitation force is quite small (i.e., linear case). Besides, it can be seen that (1) in Figure 5(a) the higher the excitation force, the wider the absorption peak due to the panel vibration and (2) in Figure 5(b) if the panel vibration is linear (or low excitation level), the absorption peak is very narrow and not very useful for widening the absorption bandwidth. Figures 6(a) and 6(b) present the sound absorption plotted against the excitation frequency for various damping ratios. The "jump down" solutions are shown in Figure 6(a) while the "jump up" solutions are in Figure 6(b). The absorption peak due to the microperforation effect is not significantly affected by the panel damping ratio. Besides, it can be seen that, (1) in Figure 6(a), the higher the damping ratio, the narrower the absorption peak due to the "jump down" panel vibration and the lower the "jump down" frequency and, (2) in Figure 6(b), the absorption peak due to the "jump up" panel vibration is not significantly affected by the damping ratio.

Figures 7(a) and 7(b) present the sound absorption and vibration amplitude plotted against the excitation frequency. Note that this case does not consider any perforation effect and other effects. There are two peaks in each of the two figures, which are due to the $(1,1)$ and $(1,3)$ mode resonances, respectively. It can be seen that the peaks due to the $(1,1)$ mode resonance are more nonlinear. As the two peaks are far from each other, no strong mode coupling effect is induced.
In Figure 7(b) the vibration amplitude is almost linearly increasing from $100 \mathrm{~Hz}$ to $286 \mathrm{~Hz}$ while the sound absorption is almost linearly increasing from $100 \mathrm{~Hz}$ to $150 \mathrm{~Hz}$ only and slowly and nonlinearly increasing from $150 \mathrm{~Hz}$ to $286 \mathrm{~Hz}$. Besides, the absorption peak due to the $(1,3)$ mode resonance is much more significant than the vibration peak. It can be implied that higher mode responses are important for absorption but not for vibration.

Figures 8(a) and 8(b) present the sound absorption plotted against the excitation frequency and show the effects of air pumping (Helmholtz resonance) and microperforation. In Figure 8(a), the peak frequencies of air pumping and microperforation are around $100 \mathrm{~Hz}$. It can be seen that the absorption bandwidth of the case with both effects is much wider and its peak absorption is much higher than the cases with air pumping or microperforation only. The two effects can interact with each other and significantly enhance the absorption performance. In Figure 8(b), the peak frequencies of air pumping and microperforation are quite far from each other. The enhancement of the absorption performance due to the two effects is not as good as that in Figure 8(a).

Figures 9(a) and 9(b) present the sound absorption plotted against the excitation frequency for various damping ratios and show the effect of linear wall vibration. The peak frequencies of wall panel and microperforation are around $80 \mathrm{~Hz}$ and $178 \mathrm{~Hz}$, respectively. It can be seen that the wall vibration does not affect the absorption performance significantly and just induces an abrupt change around $80 \mathrm{~Hz}$. The magnitude of the abrupt change depends on the damping ratio (i.e., the higher the damping ratio, the smaller the abrupt change). Figures 10(a) and 10(b) present the sound absorption plotted against the excitation frequency and show the effects of microperforation and linear and nonlinear vibrations. It can be seen that the effects of microperforation and nonlinear panel vibration are positive and significant while the effect of linear wall vibration is negative but not significant.

\section{Conclusions}

In this study, the analytic absorption formulation has been developed for the panel absorber under the effects of microperforation, air pumping, and linear and nonlinear vibration. From the predictions, it can be concluded that (1) if the panel resonant frequency is higher than the absorption peak frequency due to the microperforation effect, the panel vibration can enhance and widen the absorption bandwidth; (2) if the panel resonant frequency is close to or lower than the absorption peak frequency, the panel vibration degrades the absorption performance or the peak absorption is deteriorated; (3) the higher the excitation force, the wider the absorption peak due to the panel vibration; (4) if the panel vibration is linear (or low excitation level), the absorption peak is very narrow and not very useful for widening the absorption bandwidth; (5) the absorption bandwidth of the case with the air pumping and microperforation effects is much wider and its peak absorption is much higher than the cases with any one of these two effects. If the peak frequencies of air pumping and microperforation are far from 


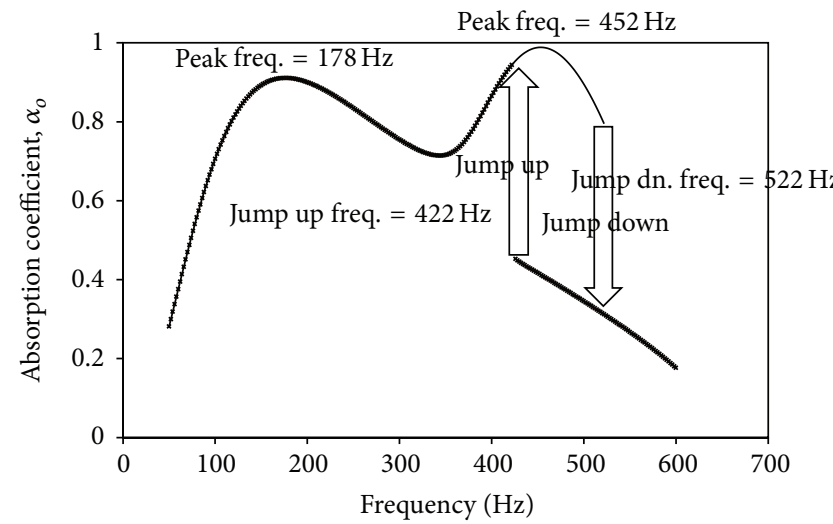

- Low to high freq. xxxxx High to low freq.

(a)

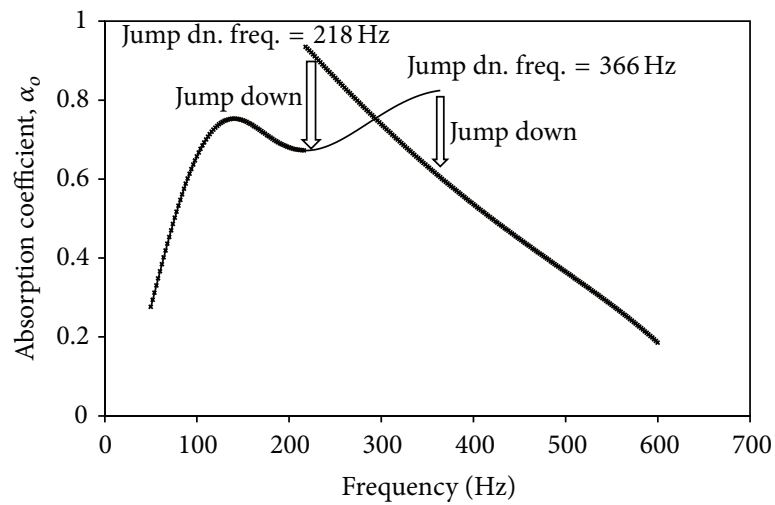

Low to high freq.

xxxxx High to low freq.

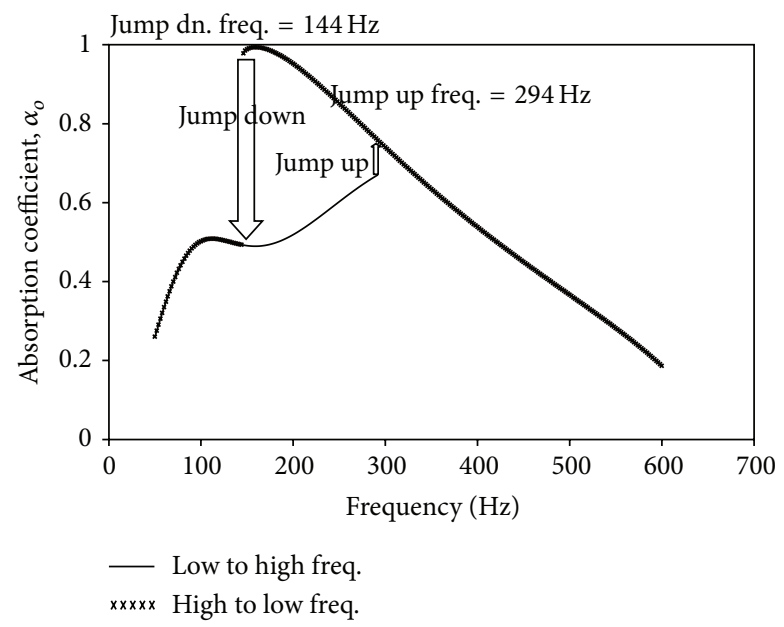

(c)

Figure 4: (a) The effect of the (1,1) mode vibration $\left(\omega_{o}=387.25 \times 2 \pi \mathrm{Hz}, t=0.8 \mathrm{~mm}, a=b=0.1 \mathrm{~m}, D=250 \mathrm{~mm}, \xi=0.01, \sigma=0.5 \%\right.$, $d=0.4 \mathrm{~mm}$, and $\kappa=10)$. (b) The effect of the (1,1) mode vibration $\left(\omega_{o}=172.11 \times 2 \pi \mathrm{Hz}, t=0.8 \mathrm{~mm}, a=b=0.15 \mathrm{~m}, D=250 \mathrm{~mm}, \xi=0.01\right.$, $\sigma=0.5 \%, d=0.4 \mathrm{~mm}$, and $\kappa=10)$. (c) The effect of the (1,1) mode vibration $\left(\omega_{o}=96.81 \times 2 \pi \mathrm{Hz}, t=0.8 \mathrm{~mm}, a=b=0.2 \mathrm{~m}, D=250 \mathrm{~mm}\right.$, $\xi=0.01, \sigma=0.5 \%, d=0.4 \mathrm{~mm}$, and $\kappa=10)$.

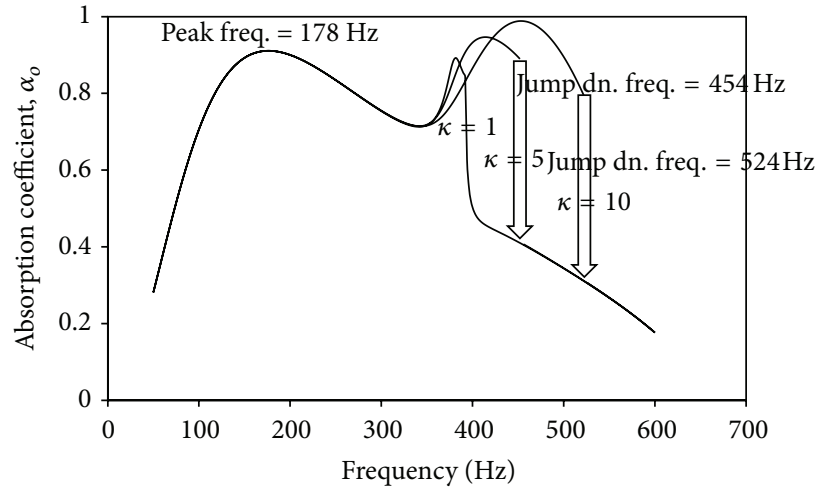

(a)

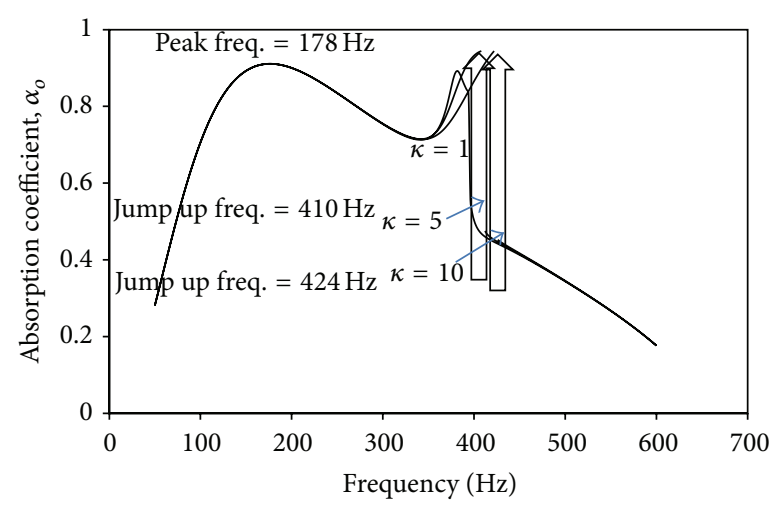

(b)

Figure 5: (a) The effect of excitation level (low to high frequency excitation, $\omega_{o}=387.25 \times 2 \pi \mathrm{Hz}, t=0.8 \mathrm{~mm}, a=b=0.1 \mathrm{~m}, D=250 \mathrm{~mm}$, $\xi=0.01, \sigma=0.5 \%$, and $d=0.4 \mathrm{~mm}$ ). (b) The effect of excitation level (high to low frequency excitation, $\omega_{o}=387.25 \times 2 \pi \mathrm{Hz}, t=0.8 \mathrm{~mm}$, $a=b=0.1 \mathrm{~m}, D=250 \mathrm{~mm}, \xi=0.01, \sigma=0.5 \%$, and $d=0.4 \mathrm{~mm})$. 


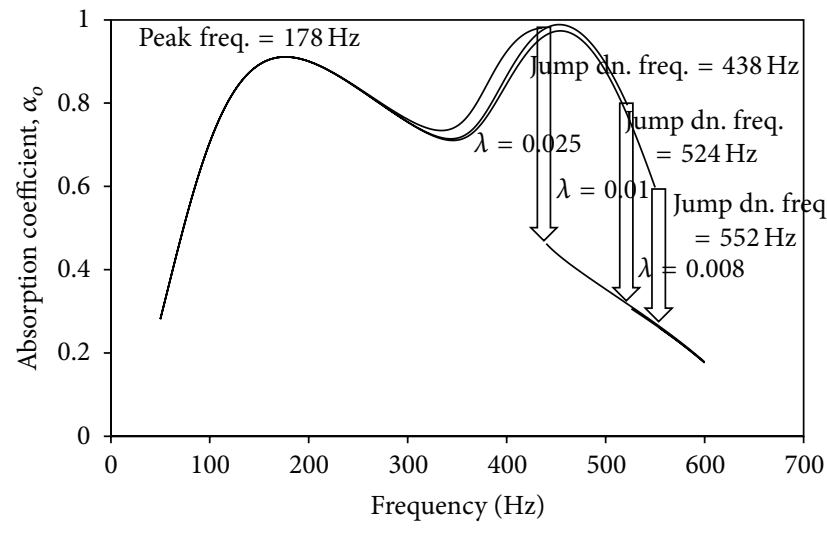

(a)

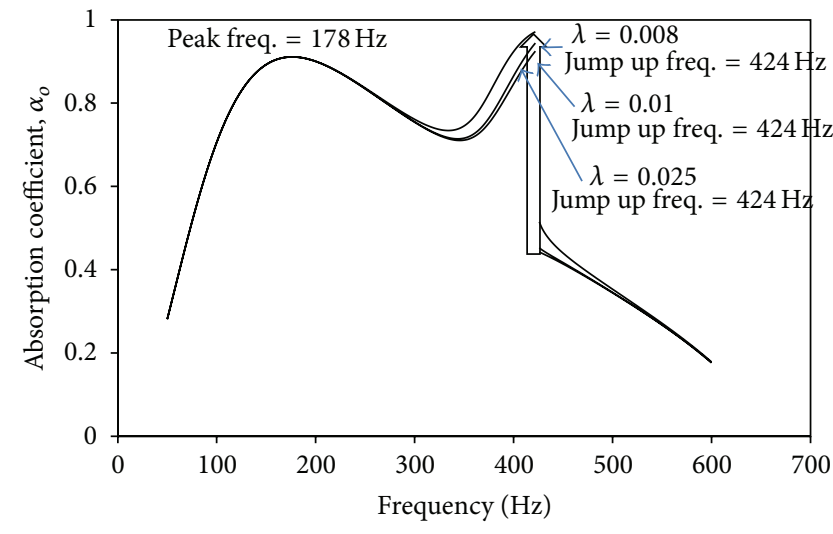

(b)

FiguRE 6: (a) The effect of damping ratio (low to high frequency excitation, $\omega_{o}=387.25 \times 2 \pi \mathrm{Hz}, t=0.8 \mathrm{~mm}, a=b=0.1 \mathrm{~m}, D=250 \mathrm{~mm}$, $\sigma=0.5 \%, d=0.4 \mathrm{~mm}$, and $\kappa=10$ ). (b) The effect of damping ratio (high to low frequency excitation, $\omega_{o}=387.25 \times 2 \pi \mathrm{Hz}, t=0.8 \mathrm{~mm}$, $a=b=0.1 \mathrm{~m}, D=250 \mathrm{~mm}, \sigma=0.5 \%, d=0.4 \mathrm{~mm}$, and $\kappa=10)$.

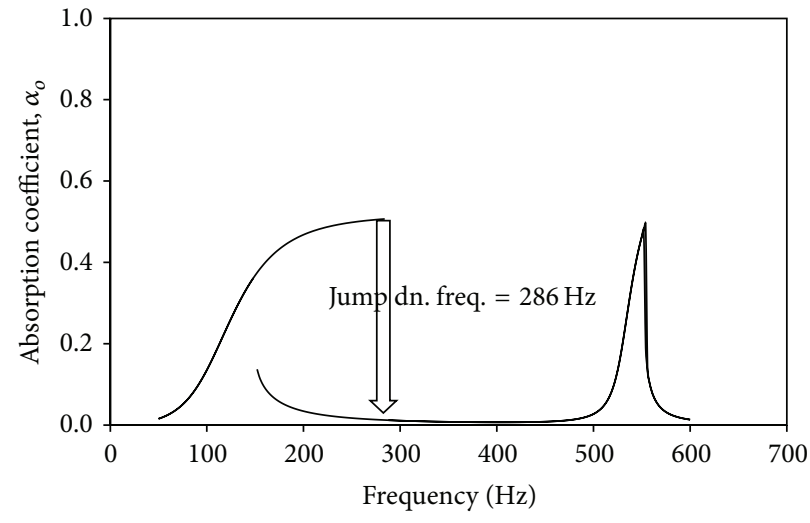

(a)

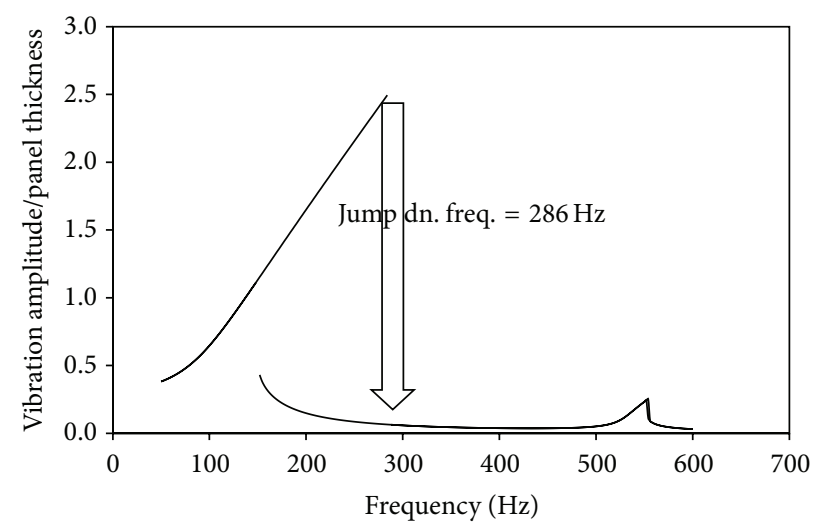

(b)

Figure 7: (a) The absorption peaks due to the $(1,1)$ and $(1,3)$ mode vibrations (low to high frequency excitation, $t=0.5 \mathrm{~mm}, a=b=0.15 \mathrm{~m}$, $D=250 \mathrm{~mm}$, and $k=5$ ). (b) The vibration peaks due to the (1,1) and (1,3) mode vibrations (low to high frequency excitation, $t=0.5 \mathrm{~mm}$, $a=b=0.15 \mathrm{~m}, D=250 \mathrm{~mm}$, and $\kappa=5)$.

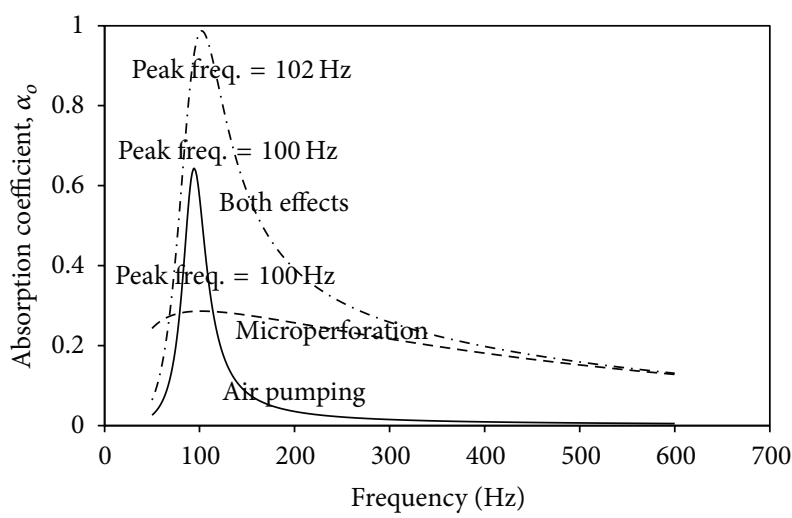

(a)

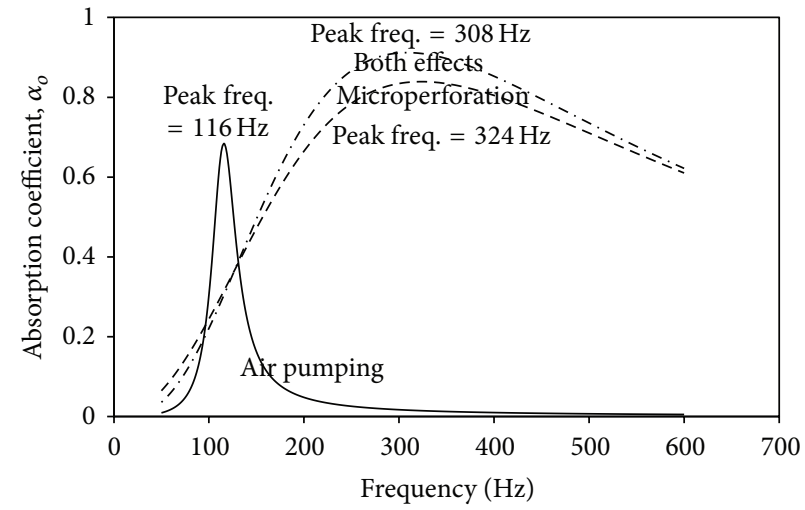

(b)

Figure 8: (a) The effects of the air pumping and microperforation $(t=0.3 \mathrm{~mm}, a=b=0.1 \mathrm{~m}, D=150 \mathrm{~mm}, \sigma=0.5 \%, d=0.3 \mathrm{~mm}$, $L=10 \mathrm{~mm}$, and $\left.a_{t}=5 \mathrm{~mm}\right)$. (b) The effects of the air pumping and microperforation $(t=0.3 \mathrm{~mm}, a=b=0.1 \mathrm{~m}, D=100 \mathrm{~mm}, \sigma=0.28 \%$, $d=0.3 \mathrm{~mm}, L=10 \mathrm{~mm}$, and $a_{t}=5 \mathrm{~mm}$ ). 


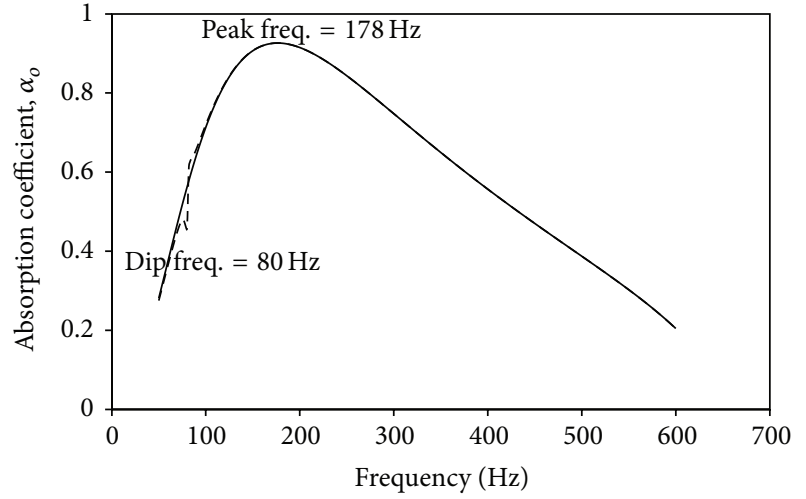

(a)

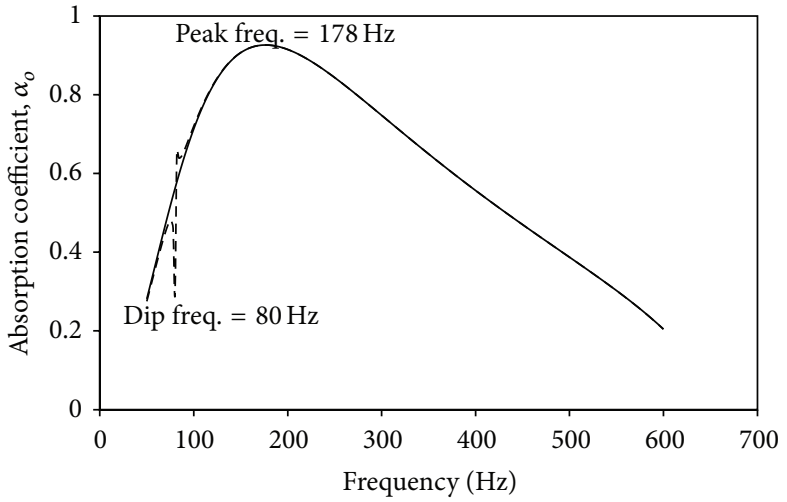

(b)

FIgURE 9: (a) The effects of linear wall vibration and microperforation $\left(\omega_{W}=80 \times 2 \pi \mathrm{Hz}, \rho_{w}=240 / \mathrm{m}^{2}, t=0.8 \mathrm{~mm}, a=b=0.1 \mathrm{~m}\right.$, $D=250 \mathrm{~mm}, \xi_{W}=0.02, \sigma=0.5 \%$, and $\left.d=0.4 \mathrm{~mm}\right)$. (b) The effects of linear wall vibration and microperforation $\left(\omega_{W}=80 \times 2 \pi \mathrm{Hz}\right.$, $\rho_{w}=240 / \mathrm{m}^{2}, t=0.8 \mathrm{~mm}, a=b=0.1 \mathrm{~m}, D=250 \mathrm{~mm}, \xi_{W}=0.01, \sigma=0.5 \%$, and $d=0.4 \mathrm{~mm}$ ).

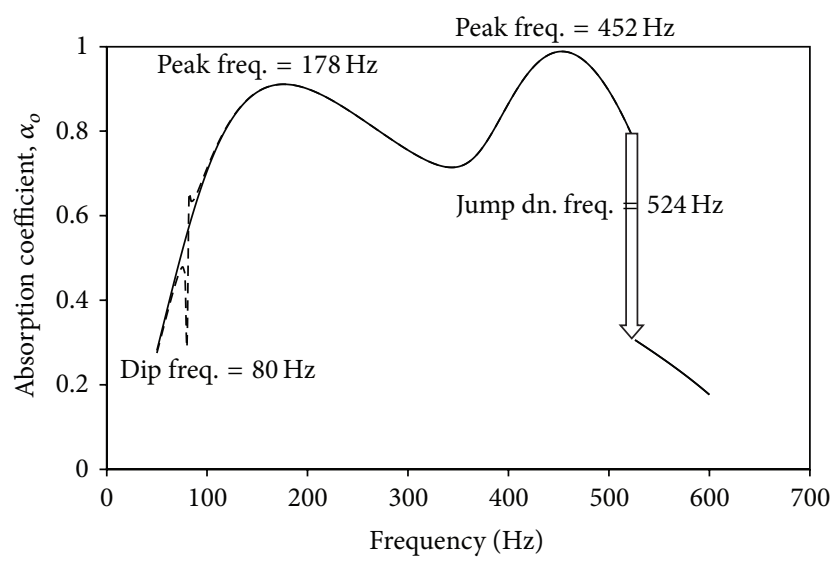

(a)

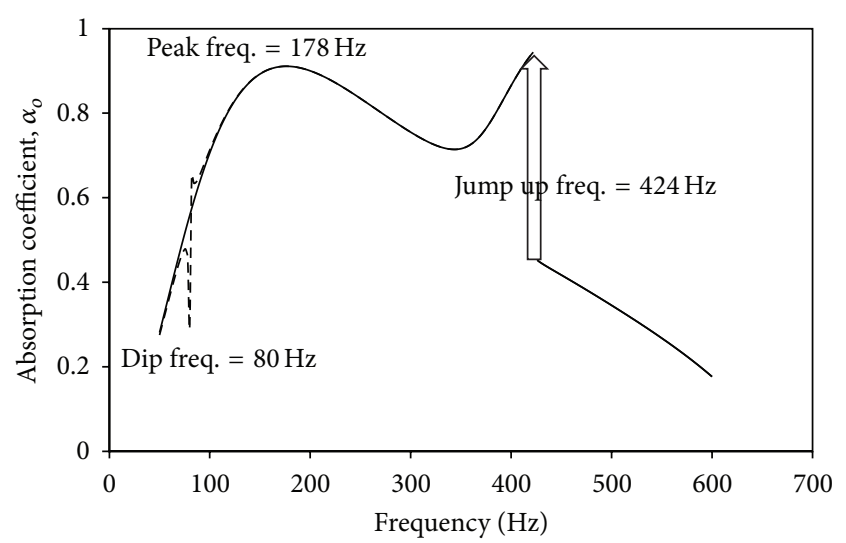

(b)

FiguRE 10: (a) The effects of microperforation and linear and nonlinear vibrations (low to high frequency excitation, $\omega_{o}=387.25 \times 2 \pi \mathrm{Hz}$, $\omega_{W}=80 \times 2 \pi \mathrm{Hz}, \rho_{w}=240 \mathrm{~kg} / \mathrm{m}^{2}, t=0.8 \mathrm{~mm}, a=b=0.1 \mathrm{~m}, D=250 \mathrm{~mm}, \xi=\xi_{W}=0.01, \sigma=0.5 \%, d=0.4 \mathrm{~mm}$, and $\left.\kappa=10\right)$. (b) The effects of microperforation and linear and nonlinear vibrations (high to low frequency excitation, $\omega_{o}=387.25 \times 2 \pi \mathrm{Hz}, \omega_{W}=80 \times 2 \pi \mathrm{Hz}$, $\rho_{w}=240 \mathrm{~kg} / \mathrm{m}^{2}, t=0.8 \mathrm{~mm}, a=b=0.1 \mathrm{~m}, D=250 \mathrm{~mm}, \xi=\xi_{W}=0.01, \sigma=0.5 \%, d=0.4 \mathrm{~mm}$, and $\left.\kappa=10\right)$.

each other, the enhancement of the absorption performance is small; and (6) the wall vibration does not affect the absorption performance significantly and just induces a small abrupt change on the absorption bandwidth around the wall resonant frequency.

\section{Appendices}

\section{A. Derivation of the Nonlinear Differential Equation}

The explanation of how the formulation of a nonlinear panel is expressed into the form of Duffing equation, which is abstracted from [30], is shown here. The flexible panel vibration is governed by the Von-Karman plate theory. The well-known partial differential equation of the nonlinear panel can be presented in terms of its displacement and the Airy stress function as follows:

$$
\begin{aligned}
& \frac{1}{12}\left(\frac{d^{2} w}{d \xi^{2}}+\nabla^{4} w\right) \\
& =\left(\frac{\partial^{2} \bar{F}}{\partial \eta^{2}} \frac{\partial^{2} w}{\partial \xi^{2}}+\frac{\partial^{2} \bar{F}}{\partial \xi^{2}} \frac{\partial^{2} w}{\partial \eta^{2}}-2 \frac{\partial^{2} \bar{F}}{\partial \eta \partial \xi} \frac{\partial^{2} w}{\partial \eta \partial \xi}\right) .
\end{aligned}
$$

Note that (A.1) is abstracted [30] and the notations are the same as those in [30] but the definitions of some notations are different from those in the main text (see the following definitions). $w=$ panel displacement; $\xi$ and $\eta$ are the dimensionless space variables of the $x$ and $y$ directions; $\xi$ is the dimensionless time variable; $v$ is Poisson's ratio. $\bar{F}$ is the Airy stress function. According to [30], the derivatives of $\bar{F}$ can be expressed in terms of the in-plane displacements 
along the $x$ and $y$ directions (i.e., $u$ and $v$ ) and transverse displacement (i.e., $w$ ).Consider

$$
\begin{aligned}
\frac{\partial^{2} \bar{F}}{\partial \xi^{2}} & =\frac{\partial v}{\partial \eta}+\frac{1}{2}\left(\frac{\partial w}{\partial \eta}\right)^{2}+v\left[\frac{\partial u}{\partial \xi}+\frac{1}{2}\left(\frac{\partial w}{\partial \xi}\right)^{2}\right], \\
\frac{\partial^{2} \bar{F}}{\partial \eta^{2}} & =\frac{\partial u}{\partial \xi}+\frac{1}{2}\left(\frac{\partial w}{\partial \xi}\right)^{2}+v\left[\frac{\partial v}{\partial \eta}+\frac{1}{2}\left(\frac{\partial w}{\partial \eta}\right)^{2}\right], \\
-\frac{\partial^{2} \bar{F}}{\partial \xi \partial \eta} & =\frac{1-v}{2}\left[\frac{\partial u}{\partial \eta}+\frac{\partial v}{\partial \xi}+\frac{\partial w}{\partial \eta} \frac{\partial w}{\partial \xi}\right] .
\end{aligned}
$$

As it is assumed that the panel is simply supported, the following displacement functions which can satisfy the boundary conditions are adopted:

$$
\begin{aligned}
w & =\varepsilon \sin (m \pi \xi) \sin (n \pi r \eta), \\
u & =\frac{\varepsilon^{2} \pi}{16}\left(\cos (2 \pi n r \eta)-1+v \frac{n^{2} r^{2}}{m^{2}}\right) m \sin (2 \pi m \xi) \\
v & =\frac{\varepsilon^{2} \pi}{16}\left(n r \cos (2 \pi m \xi)-n r+v \frac{m^{2}}{n r}\right) \sin (2 \pi n r \eta) .
\end{aligned}
$$

Again, (A.3a)-(A.3c) originate from [30] and the definitions of some notations are different from those in the main text (see the following definitions). $m$ and $n$ are the modal number. $\varepsilon$ is the modal amplitude. Consider $r=a / b$.

Then, consider putting (A.2a)-(A.2c) and (A.3a)-(A.3c) into the right terms of (A.1):

$$
\begin{aligned}
& \frac{\partial^{2} \bar{F}}{\partial \xi^{2}} \frac{\partial^{2} w}{\partial \eta^{2}} \\
& =-\frac{\partial^{2} \bar{F}}{\partial \xi^{2}} \pi^{2} n^{2} r^{2} M \sin (m \pi \xi) \sin (\pi n r \eta) \\
& =-\frac{\varepsilon^{3} \pi^{4}}{8} n^{4} r^{4}\left(\cos (2 \pi m \xi)-1+\nu \frac{m^{2}}{n^{2} r^{2}}\right) \\
& \quad \times \cos (2 \pi n r \eta) \sin (m \pi \xi) \sin (\pi n r \eta) \\
& \quad-\frac{1}{2} \varepsilon^{3} \pi^{4} n^{4} r^{4} \sin { }^{2}(\pi m \xi) \\
& \quad \times \cos { }^{2}(\pi n r \eta) \sin (m \pi \xi) \sin (\pi n r \eta) \\
& \quad-\varepsilon^{3} \pi^{4} n^{2} r^{2} m^{2}\left(\cos (2 \pi r \eta)-1+\nu \frac{n^{2} r^{2}}{m^{2}}\right) \\
& \quad \times \cos (2 \pi m \xi) \sin (m \pi \xi) \sin (\pi n r \eta)
\end{aligned}
$$

$$
\begin{aligned}
& -\nu \frac{1}{2} \varepsilon^{3} \pi^{4} m^{2} n^{2} r^{2} \\
& \times \cos ^{2}(\pi m \xi) \sin ^{2}(\pi n r \eta) \sin (m \pi \xi) \sin (\pi n r \eta),
\end{aligned}
$$

$$
\begin{aligned}
& \frac{\partial^{2} \bar{F}}{\partial \eta^{2}} \frac{\partial^{2} w}{\partial \xi^{2}} \\
& =-\frac{\partial^{2} \bar{F}}{\partial \eta^{2}} m^{2} \pi^{2} \varepsilon \sin (\pi m \xi) \sin (\pi n r \eta) \\
& =-\frac{\varepsilon^{3} \pi^{4}}{8} m^{4}\left(\cos (2 \pi r \eta)-1+v \frac{n^{2} r^{2}}{m^{2}}\right) \\
& \times \cos (2 \pi m \xi) \sin (\pi m \xi) \sin (\pi n r \eta)-\frac{1}{2} \varepsilon^{3} \pi^{4} m^{4} \\
& \times \cos ^{2}(\pi m \xi) \sin ^{2}(\pi n r \eta) \sin (\pi m \xi) \sin (\pi n r \eta) \\
& -\nu \frac{\varepsilon^{3} \pi^{4}}{8} n^{2} r^{2} m^{2}\left(\cos (2 \pi m \xi)-1+v \frac{m^{2}}{n^{2} r^{2}}\right) \\
& \times \cos (2 \pi n r \eta) \sin (\pi m \xi) \sin (\pi n r \eta) \\
& -\nu \frac{1}{2} \varepsilon^{3} \pi^{4} m^{2} n^{2} r^{2} \sin ^{2}(\pi m \xi) \\
& \times \cos ^{2}(\pi n r \eta) \sin (\pi m \xi) \sin (\pi n r \eta) . \\
& -\frac{\partial^{2} \bar{F}}{\partial \xi \partial \eta} \\
& =\frac{1-v}{2}\left[\frac{\partial u}{\partial \eta}+\frac{\partial v}{\partial \xi}+\frac{\partial w}{\partial \eta} \frac{\partial w}{\partial \xi}\right] \\
& =\frac{1-\nu}{2}\left[-\frac{\varepsilon^{2} m n r \pi^{2}}{4} \sin (2 \pi n r \eta) \sin (2 \pi m \xi)\right. \\
& +\varepsilon^{2} m n r \pi^{2} \sin (\pi n r \eta) \\
& \times \cos (\pi n r \eta) \sin (\pi m \xi) \cos (\pi m \xi)]=0 .
\end{aligned}
$$

Hence, (A.1) can be rewritten in the following form:

$$
\begin{gathered}
{\left[C_{1} \frac{d^{2} \varepsilon}{d \xi^{2}}+C_{2} \varepsilon+C_{3} \varepsilon^{3}\right] \sin (\pi m \xi) \sin (\pi n r \eta)} \\
+ \text { higher mode terms }=0,
\end{gathered}
$$

where $C_{1}, C_{2}$, and $C_{3}$ are the constants dependent of $m$, $n, r, v$, and the material and dimension parameters and so forth. According to [30], the higher mode terms in (A.5) are neglected. For forced vibration cases, a forcing term $P$ is considered. That is,

$$
C_{1} \frac{d^{2} \varepsilon}{d \xi^{2}}+C_{2} \varepsilon+C_{3} \varepsilon^{3}+P=0
$$


where (A.6) is a Duffing equation which form is the same as that of (1).

\section{B. Derivation of the Peak Frequency}

Consider a damping term and rewrite (1):

$$
\rho \frac{d^{2} \varepsilon}{d \tau^{2}}+2 \xi \rho \omega_{o} \frac{d \varepsilon}{d \tau}+\rho \omega_{o}^{2} \varepsilon+\beta \varepsilon^{3}+F_{o, m n} \sin (\omega \tau)=0 .
$$

Take the approximation of $\varepsilon=A_{\cos } \cos (\omega t)+A_{\sin } \sin (\omega t)$ and consider the harmonic balance of $\sin (\omega t)$ and $\cos (\omega t)$ :

$$
\begin{gathered}
-\rho \omega^{2} A_{\text {sin }}-2 \xi \rho \omega_{o} \omega A_{\mathrm{cos}}+\rho \omega_{o}^{2} A_{\text {sin }} \\
+\frac{3}{4} \beta A_{\mathrm{sin}}^{3}+\frac{3}{4} \beta A_{\sin } A_{\mathrm{cos}}^{2}+F_{o, m n}=0, \\
-\rho \omega^{2} A_{\mathrm{cos}}+2 \xi \rho \omega_{o} \omega A_{\mathrm{sin}}+\rho \omega_{o}^{2} A_{\mathrm{cos}} \\
+\frac{3}{4} \beta_{c} A_{\cos }^{3}+\frac{3}{4} \beta_{c} A_{\sin }^{2} A_{\mathrm{cos}}=0 .
\end{gathered}
$$

Let $A_{o}^{2}=A_{\cos }^{2}+A_{\sin }^{2}$. Multiplying (B.2a) and (B.2b) by $A_{\cos }$ and $A_{\text {sin }}$, respectively, and then subtracting the new (B.2a) by the new (B.2b) yield

$$
\begin{gathered}
-\frac{2 \xi \rho \omega_{o} \omega A_{o}^{2}}{F_{o, m n}}=A_{\text {cos }}, \\
A_{o} \sqrt{1-\left(\frac{2 \xi \rho \omega_{o} \omega A_{o}}{F_{o, m n}}\right)^{2}}=A_{\sin } .
\end{gathered}
$$

Then inputting (B.3a)-(B.3b) into (B.2b) yields

$$
\begin{aligned}
& \rho\left(\omega^{2}-\omega_{o}^{2}\right) \frac{2 \xi \rho \omega_{o} \omega A_{o}^{2}}{F_{o, m n}}+2 \xi \rho \omega_{o} \omega A_{o} \sqrt{1-\left(\frac{2 \xi \rho \omega_{o} \omega A_{o}}{F_{o, m n}}\right)^{2}} \\
& -\frac{3}{4} \beta\left(A_{o}\right)^{4} \frac{2 \xi \rho \omega_{o} \omega}{F_{o, m n}}=0 .
\end{aligned}
$$

Consider the equation representing the backbone curve by taking the forcing and damping terms out of (B.1):

$$
\begin{gathered}
\rho\left(-\omega^{2}+\omega_{o}^{2}\right) A_{o}+\frac{3}{4} \beta A_{o}^{3}=0, \\
A_{o}^{2}=\frac{\rho\left(\omega^{2}-\omega_{o}^{2}\right)}{(3 / 4) \beta} .
\end{gathered}
$$

Inputting (B.6) into (B.4) yields

$$
1-\left(\frac{2 \xi \rho \omega_{o} \omega}{F_{o, m n}}\right)^{2} \frac{\rho\left(\omega^{2}-\omega_{o}^{2}\right)}{(3 / 4) \beta}=0,
$$

where (B.7) contains only one unknown, $\omega$. It is obtainable and represents the frequency at which the vibration amplitude peaks and is equal to $\omega_{N}$ in (9).

\section{Conflict of Interests}

The author declares that there is no conflict of interests regarding the publication of this paper.

\section{Acknowledgments}

The work in this paper was fully supported by the CityU grant "Nonlinear Dropped Ceiling for Noise and Vibration Reduction, Project no. 7004039." The author would like to express the sincere gratitude and appreciation to Dr. WY Poon for his advice about this research.

\section{References}

[1] Y. Y. Lee and C. F. Ng, "Sound insertion loss of stiffened enclosure plates using the finite element method and the classical approach," Journal of Sound and Vibration, vol. 217, no. 2, pp. 239-260, 1998.

[2] Y. Y. Lee, "The response frequency conversion characteristic of a nonlinear curved panel with a centre mass and the sound radiations," Mathematical Problems in Engineering, vol. 2012, Article ID 298413, 11 pages, 2012.

[3] S. Y. Hsia and Y. T. Chou, "Traffic noise propagating from vibration of railway wagon," Mathematical Problems in Engineering, vol. 2013, Article ID 651797, 7 pages, 2013.

[4] J. Feng, X. P. Zheng, H. T. Wang, Y. J. Zou, Y. H. Liu, and Z. H. Yao, "Low-frequency acoustic-structure analysis using coupled FEM-BEM method," Mathematical Problems in Engineering, vol. 2013, Article ID 583079, 8 pages, 2013.

[5] S. M. Chen, D. F. Wang, and J. M. Zan, "Interior noise prediction of the automobile based on hybrid FE-SEA method," Mathematical Problems in Engineering, vol. 2011, Article ID 327170, 20 pages, 2011.

[6] R. V. Craster and S. G. L. Smith, "A class of expansion functions for finite elastic plates in structural acoustics," Journal of the Acoustical Society of America, vol. 106, no. 6, pp. 3128-3134, 1999.

[7] K. A. Mulholland and H. D. Parbrook, "Transmission of sound through apertures of negligible thickness," Journal of Sound and Vibration, vol. 5, no. 3, pp. 499-508, 1967.

[8] R. D. Ford and M. A. McCormick, "Panel sound absorbers," Journal of Sound and Vibration, vol. 10, no. 3, pp. 411-423, 1969.

[9] D. Maa, "Microperforated-panel wideband absorbers," Noise Control Engineering Journal, vol. 29, no. 3, pp. 77-84, 1987.

[10] D. Maa, "Potential of microperforated panel absorber," Journal of the Acoustical Society of America, vol. 104, no. 5, pp. 28612866, 1998.

[11] Y. Wu and G. Han, "Infinitely many sign-changing solutions for some nonlinear fourth-order beam equations," Abstract and Applied Analysis, vol. 2013, Article ID 635265, 11 pages, 2013.

[12] X. Liu, B. Huo, and S. Zhang, "Nonlinear dynamic analysis on the rain-wind-induced vibration of cable considering the equilibrium position of rivulet," Abstract and Applied Analysis, vol. 2013, Article ID 927632, 10 pages, 2013.

[13] X. Lin and F. Li, "Asymptotic energy estimates for nonlinear Petrovsky plate model subject to viscoelastic damping," Abstract and Applied Analysis, vol. 2012, Article ID 419717, 25 pages, 2012.

[14] J. A. Esquivel-Avila, "Dynamic analysis of a nonlinear Timoshenko equation," Abstract and Applied Analysis, vol. 2011, Article ID 724815, 36 pages, 2011. 
[15] M. L. Santos, J. Ferreira, and C. A. Raposo, "Existence and uniform decay for a nonlinear beam equation with nonlinearity of Kirchhoff type in domains with moving boundary," Abstract and Applied Analysis, no. 8, pp. 901-919, 2005.

[16] X. He, "Modal decoupling using the method of weighted residuals for the nonlinear elastic dynamics of a clamped laminated composite," Mathematical Problems in Engineering, vol. 2009, Article ID 972930, 19 pages, 2009.

[17] D. Wang, J. Zhang, and Y. Wang, "Strong attractor of beam equation with structural damping and nonlinear damping," Mathematical Problems in Engineering, vol. 2013, Article ID 769514, 8 pages, 2013.

[18] Y. Y. Lee, "Structural-acoustic coupling effect on the nonlinear natural frequency of a rectangular box with one flexible plate," Applied Acoustics, vol. 63, no. 11, pp. 1157-1175, 2002.

[19] Y. Y. Lee, "Analysis of the nonlinear structural-acoustic resonant frequencies of a rectangular tube with a flexible end using harmonic balance and homotopy perturbation methods," Abstract and Applied Analysis, vol. 2012, Article ID 391584, 13 pages, 2012.

[20] C. K. Hui, Y. Y. Lee, and J. N. Reddy, "Approximate elliptical integral solution for the large amplitude free vibration of a rectangular single mode plate backed by a multi-acoustic mode cavity," Thin-Walled Structures, vol. 49, no. 9, pp. 1191-1194, 2011.

[21] Y. Y. Lee, Q. S. Li, A. Y. T. Leung, and R. K. L. Su, "The jump phenomenon effect on the sound absorption of a nonlinear panel absorber and sound transmission loss of a nonlinear panel backed by a cavity," Nonlinear Dynamics, vol. 69, no. 1-2, pp. 99116, 2012.

[22] Y. Y. Lee, R. K. L. Su, C. F. Ng, and C. K. Hui, "The effect of modal energy transfer on the sound radiation and vibration of a curved panel: theory and experiment," Journal of Sound and Vibration, vol. 324, no. 3-5, pp. 1003-1015, 2009.

[23] A. Mamandi, M. H. Kargarnovin, and S. Farsi, "Dynamic analysis of a simply supported beam resting on a nonlinear elastic foundation under compressive axial load using nonlinear normal modes techniques under three-to-one internal resonance condition," Nonlinear Dynamics, vol. 70, no. 2, pp. 1147$1172,2012$.

[24] A. H. Nayfeh, The Method of Normal Forms, Wiley-VCH, Weinheim, Germany, 2nd edition, 2011.

[25] M. H. Ghayesh and S. Balar, "Non-linear parametric vibration and stability of axially moving visco-elastic Rayleigh beams," International Journal of Solids and Structures, vol. 45, no. 25-26, pp. 6451-6467, 2008.

[26] S. W. Shaw and C. Pierre, "Normal modes of vibration for nonlinear continuous systems," Journal of Sound and Vibration, vol. 169, no. 3, pp. 319-347, 1994.

[27] M. E. King and A. F. Vakakis, "Energy-based formulation for computing nonlinear normal modes in undamped continuous systems," Journal of Vibration and Acoustics, Transactions of the ASME, vol. 116, no. 3, pp. 332-340, 1994.

[28] J. Kang and H. V. Fuchs, "Predicting the absorption of open weave textiles and micro-perforated membranes backed by an air space," Journal of Sound and Vibration, vol. 220, no. 5, pp. 905-920, 1999.

[29] L. E. Kinsler, A. R. Frey, A. B. Coppens, and J. V. Sanders, Fundamentals of Acoustics, John Wiley \& Sons, 4th edition, 1999.

[30] H. N. Chu and G. Herrmann, "Influence of large amplitudes on free flexural vibrations of rectangular elastic plates," Journal of Applied Mechanics, vol. 23, pp. 532-540, 1956.
[31] W. Weisstein Eric, "Vieta's Substitution," From MathWorld-A Wolfram Web Resource, http://mathworld.wolfram.com/VietasSubstitution.html.

[32] Y. Y. Lee, X. Guo, and E. W. M. Lee, "Effect of the large amplitude vibration of a finite flexible micro-perforated panel absorber on sound absorption," International Journal of Nonlinear Sciences and Numerical Simulation, vol. 8, no. 1, pp. 41-44, 2007.

[33] Y. Y. Lee, E. W. M. Lee, and C. F. Ng, "Sound absorption of a finite flexible micro-perforated panel backed by an air cavity," Journal of Sound and Vibration, vol. 287, no. 1-2, pp. 227-243, 2005.

[34] Y. Y. Lee and E. W. M. Lee, "Widening the sound absorption bandwidths of flexible micro-perforated curved absorbers using structural and acoustic resonances," International Journal of Mechanical Sciences, vol. 49, no. 8, pp. 925-934, 2007. 


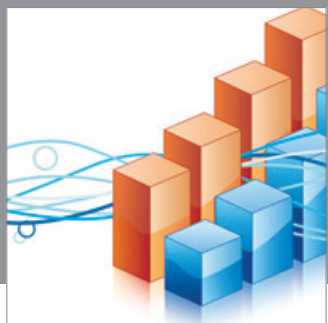

Advances in

Operations Research

mansans

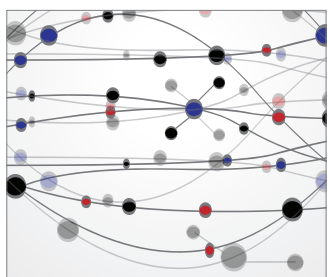

The Scientific World Journal
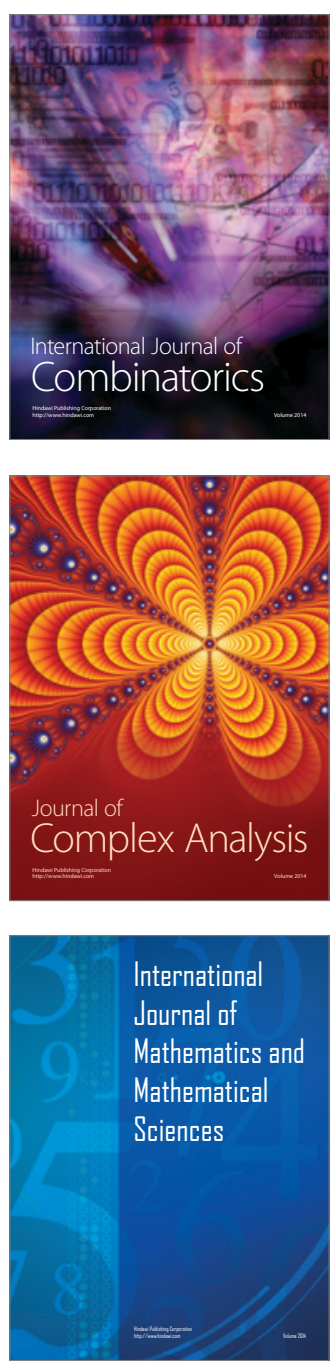
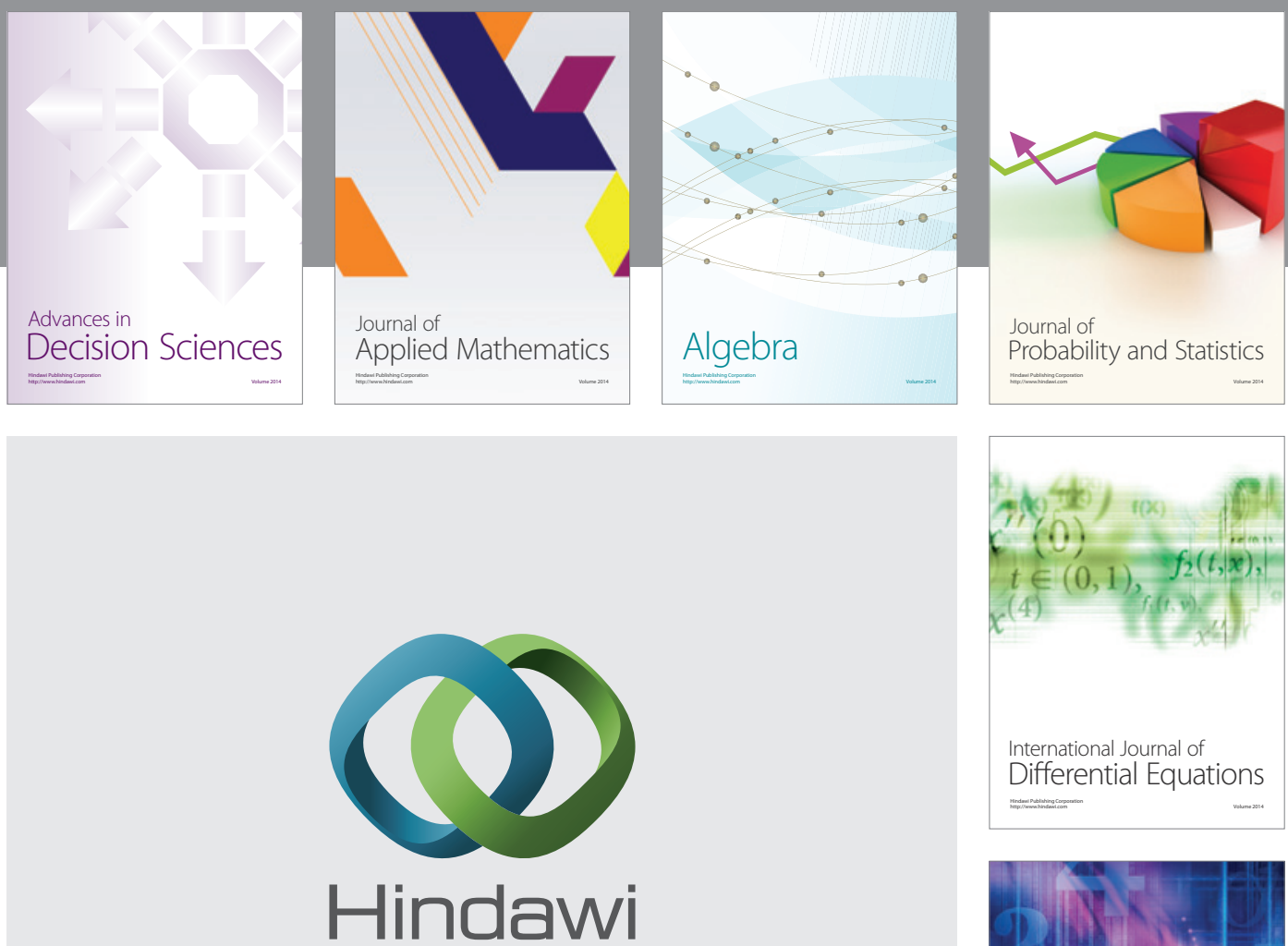

Submit your manuscripts at http://www.hindawi.com
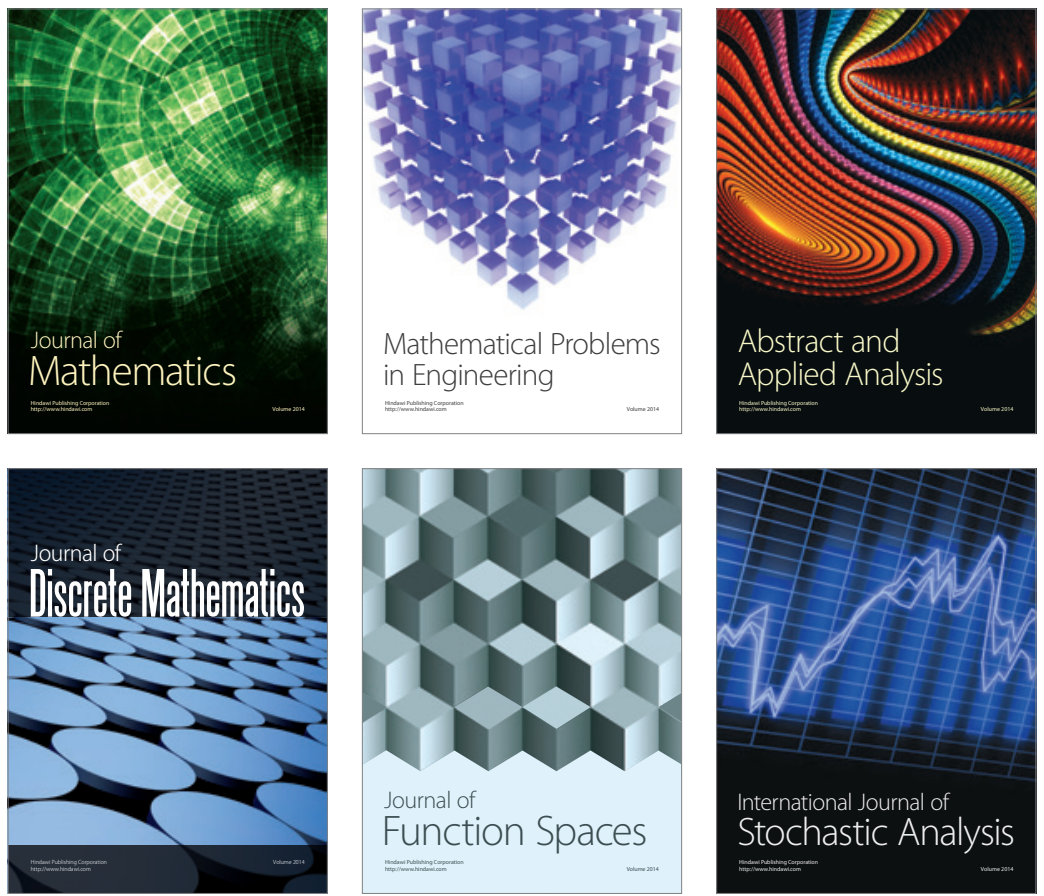

Journal of

Function Spaces

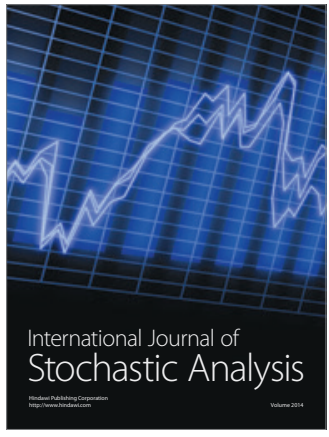

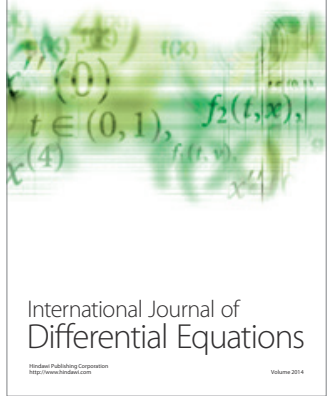
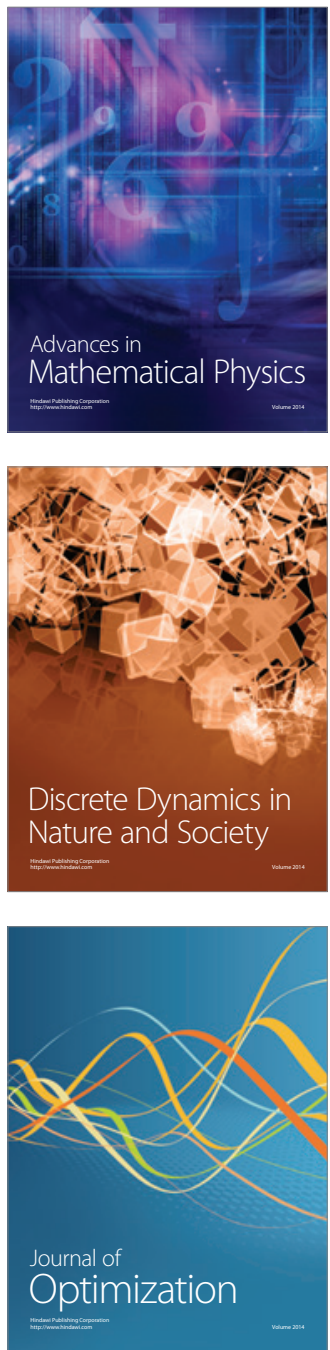